\title{
- Stochastic Representation of Chaos Using Terminal Attractors
}

\section{Fictitious control forces stabilize what would otherwise be unstable computed trajectories.}

\author{
NASA's Jet Propulsion Laboratory, Pasadena, California
}

A nonlinear version of the Liouville equation based on terminal attractors is part of a mathematical formalism for describing postinstability motions of $\mathrm{dy}$ namical systems characterized by exponential divergences of trajectories leading to chaos (including turbulence as a form of chaos). The formalism can be applied to both conservative systems (e.g., multibody systems in celestial mechanics) and dissipative systems (e.g., viscous fluids).

This formalism at an earlier stage of development was reported in "Extension of Liouville Formalism to Postinstability Dynamics" (NPO-30393), NASA Tech Briefs, Vol. 27, No. 9 (September 2003), page 56 . To recapitulate: The problem is to predict the postinstability motions of a dynamic system governed by a system of nonlinear equations and subject to initial conditions. The formalism of nonlinear dynamics does not afford means to discriminate between stable and unstable motions: an additional stability analysis is necessary for such discrimination. However, an additional stability analysis does not suggest any modifications of a mathematical model that would enable the model to describe postinstability motions efficiently. The most important type of instability that necessitates a postinstability description is associated with positive Lyapunov exponents. Such an instability leads to exponential growth of small errors in initial conditions or, equivalently, exponential divergence of neighboring trajectories.

The development of the present formalism was undertaken in an effort to remove positive Lyapunov exponents. The means chosen to accomplish this is coupling of the governing dynamical equations with the corresponding Liouville equation that describes the evolution of the flow of error probability. The underlying idea is to suppress the divergences of different trajectories that correspond to different initial conditions, without affecting a target trajectory, which is one that starts with prescribed initial conditions.

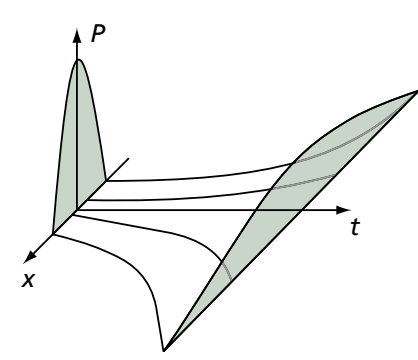

Uncontrolled Evolution

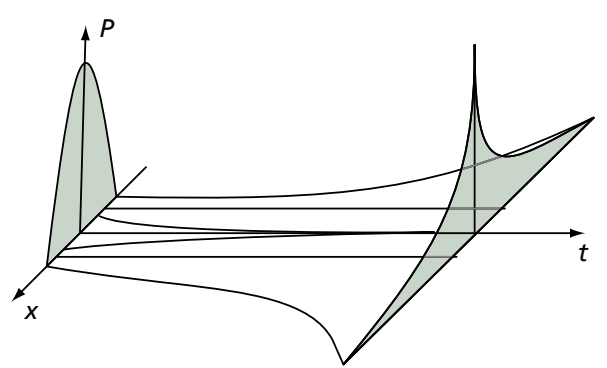

Controlled Evolution

The Probability Distribution of Error about a target trajectory becomes flattened in uncontrolled evolution as close neighboring trajectories diverge. However, when evolution is controlled by fictitious stabilizing forces that create a terminal attractor in probability space, the distribution of error becomes more sharply peaked about the target trajectory.

This formalism applies to a system of $n$ first-order ordinary differential equations in $n$ unknown dynamical (state) variables:

$$
\dot{x}_{i}=f_{i}[\mathbf{x}(t), t],
$$

where $i$ is an integer between 1 and $n, x_{i}$ is one of the unknown dynamical variables, the overdot signifies differentiation with respect to time, $\mathbf{x}$ is the vector of all the dynamical variables $\left(x_{1}, x_{2}, \ldots x_{n}\right)$, and $t$ is time. The prescribed initial conditions are given by

$$
x_{i}=x_{i}^{0},
$$

The corresponding Liouville equation for the evolution of the probability distribution, $P\left(x_{1}, x_{2}, \ldots x_{n}, t\right)$, of errors in the initial conditions is

$$
\frac{\partial P}{\partial t}+\nabla \cdot(P \mathbf{f})=0
$$

where $\mathbf{f}$ is the vector of all the forcing functions $\left(f_{1}, f_{2}, \ldots f_{n}\right)$. It is assumed that this probability distribution peaks at zero error (representing the prescribed initial conditions).

Fictitious control (stabilizing) forces $\left[\mathbf{F}=\left(F_{1}, F_{2}, \ldots F_{n}\right)\right]$ are added to the system of differential equations. The form of these forces differs from that of the fictitious stabilizing force described in the cited previous article: Whereas previously, the fictitious stabilizing force was proportional to the gradient of the probability density in the space of the dynam- ical variables, the present fictitious control forces are functions of the differences between expected and actual values of the dynamical variables $x_{i}$ :

$$
F_{i} \equiv \gamma_{i}\left(\left\langle x_{i}\right\rangle-x_{i}\right)^{\frac{1}{3}}
$$

where $\gamma_{i}$ is a positive constant and $x_{i}$ is the expected value of $x_{i}$, as given by

$$
\left\langle x_{i}\right\rangle=\int_{-\infty}^{\infty} X_{i} P d X_{1} \ldots d X_{n}
$$

The control forces have two important properties:

- Because they vanish as $\mathbf{x} \rightarrow\langle\mathbf{x}\rangle$, they do not affect the target trajectory; and

- Because the magnitudes of their derivatives approach $\infty$ as $\mathbf{x} \rightarrow\langle\mathbf{x}\rangle$, they make the target trajectory infinitely stable. In other words, the target trajectory becomes a terminal attractor.

The resulting modified system of dynamical equations is

$$
\begin{aligned}
& \dot{x}_{i}=f_{i}+\gamma_{i}\left(\left\langle x_{i}\right\rangle-x_{i}\right)^{\frac{1}{3}}
\end{aligned}
$$

The corresponding modified Liouville equation is

$$
\frac{\partial P}{\partial t}=-\sum_{i=1}^{n} \frac{\partial}{\partial x_{i}}\left\{P\left[f_{i}+\gamma_{i}\left(\left\langle x_{i}\right\rangle-x_{i}\right)^{\frac{1}{3}}\right]\right\},
$$

wherein the terminal attractors act as nonlinear sinks of probability.

At the limit as $x_{i} \rightarrow\left\langle x_{i}\right\rangle$, one can neglect the real force $f_{i}$ as being much smaller than the control force $F_{i}$, making it possible to decompose the Liouville equation 
into $n$ independent equations and to express $P$ as a product of $n$ probabilities $P_{i}$ :

$$
\begin{aligned}
& \frac{\partial P_{i}^{\prime}}{\partial t}=-\gamma_{i} \frac{\partial P_{i}^{\prime}}{\partial x_{i}}\left(\left\langle x_{i}\right\rangle-x_{i}\right)^{\frac{1}{3}} \text { and } \\
& P\left(x_{1}, x_{2} \ldots x_{n}\right)=\prod_{i=1}^{n} P_{i}^{\prime}\left(x_{i}\right)
\end{aligned}
$$

By use of these equations, it can be shown that the control forces create a powerful terminal attractor in probability space that corresponds to occur- rence of the target trajectory with probability one (see figure). In configuration space (space in the sense in which "space" is understood in casual conversation), the effect of the control forces is to suppress exponential divergence of close neighboring trajectories without affecting the target trajectory. As a result, the post-instability motion is represented by a set of functions that describe the evolution of such statistical invariants such as expectations, vari- ances, and higher moments of the statistics of the state variables $x_{i}$ as functions of time.

This work was done by Michail Zak of Caltech for NASA's Jet Propulsion Laboratory. Further information is contained in a TSP (see page 1).

The software used in this innovation is available for commercial licensing. Please contact Karina Edmonds of the California Institute of Technology at (818) 393-2827. Refer to NPO-41519.

\section{$\checkmark$ Two High-Temperature Foil Journal Bearings}

These are prototypes of foil bearings for aircraft gas turbine engines.

\section{John H. Glenn Research Center, Cleveland, Ohio}

An enlarged, high-temperature-compliant foil bearing has been built and tested to demonstrate the feasibility of such bearings for use in aircraft gas turbine engines. At $150 \mathrm{~mm}$ in diameter, this is the largest foil bearing known to date. This bearing is a scaled-up version of a patented 100-mm-diameter foil bearing, augmented by coating the foil with a proprietary high-temperature material. In a companion development, a foil bearing as described above has been combined with a 150 -mm-diameter active magnetic bearing to make a hybrid foil magnetic bearing.

Foil bearings are attractive for use in some machines in which (1) speeds of rotation, temperatures, or both exceed maximum allowable values for rollingelement bearings; (2) conventional lubricants decompose at high operating temperatures; and/or (3) it is necessary or desirable not to rely on conventional lubrication systems. In a foil bearing, the lubricant is the working fluid (e.g., air or a mixture of combustion gases) in the space between the journal and the shaft in the machine in which the bearing is installed. At no or low speed, the shaft is supported at by a spring-loaded foil journal lining. Once the shaft is rotating rapidly enough, the hydrodynamic and viscous forces exerted by the flow of working fluid between the foil and the shaft force the foil away from the shaft, so that the shaft becomes supported by a film of the working fluid.

The present enlarged, high-temperature foil bearing has been tested at speeds up to $27,000 \mathrm{rpm}$ (at $150 \mathrm{~mm}$ diameter, corresponding to a surface speed of $212 \mathrm{~m} / \mathrm{s}$ ) and at temperatures in excess of $1,200{ }^{\circ} \mathrm{F}\left(>649{ }^{\circ} \mathrm{C}\right)$. These speed and temperature limits exceed those of rolling-element bearings by several fold. conceived to take advantage of the strengths of the foil and the active magnetic bearing while utilizing each bearing to compensate for the weakness of the other, for the overall purpose of obtaining high load capacity at all speeds and temperatures (see figure). The active magnetic bearing exhibits excellent performance at low speed, where the surface coating on the foil bearing has limited load capacity. The foil bearing
The hybrid foil magnetic bearing was

exhibits excellent performance at high speed, where the active magnetic bearing can fail in response to shocks and other transient disturbances.

Unlike a conventional active magnetic bearing, the hybrid foil magnetic bearing can operate without need for a separate protective auxiliary/backup bearing. In case of failure of the active magnetic bearing in the hybrid foil magnetic bearing, the foil bearing plays the role of the backup bearing, so that a rotor can continue to run on the foil bearing alone and then come down to a safe stop. The hybrid foil magnetic bearing exhibits both the high load ca-

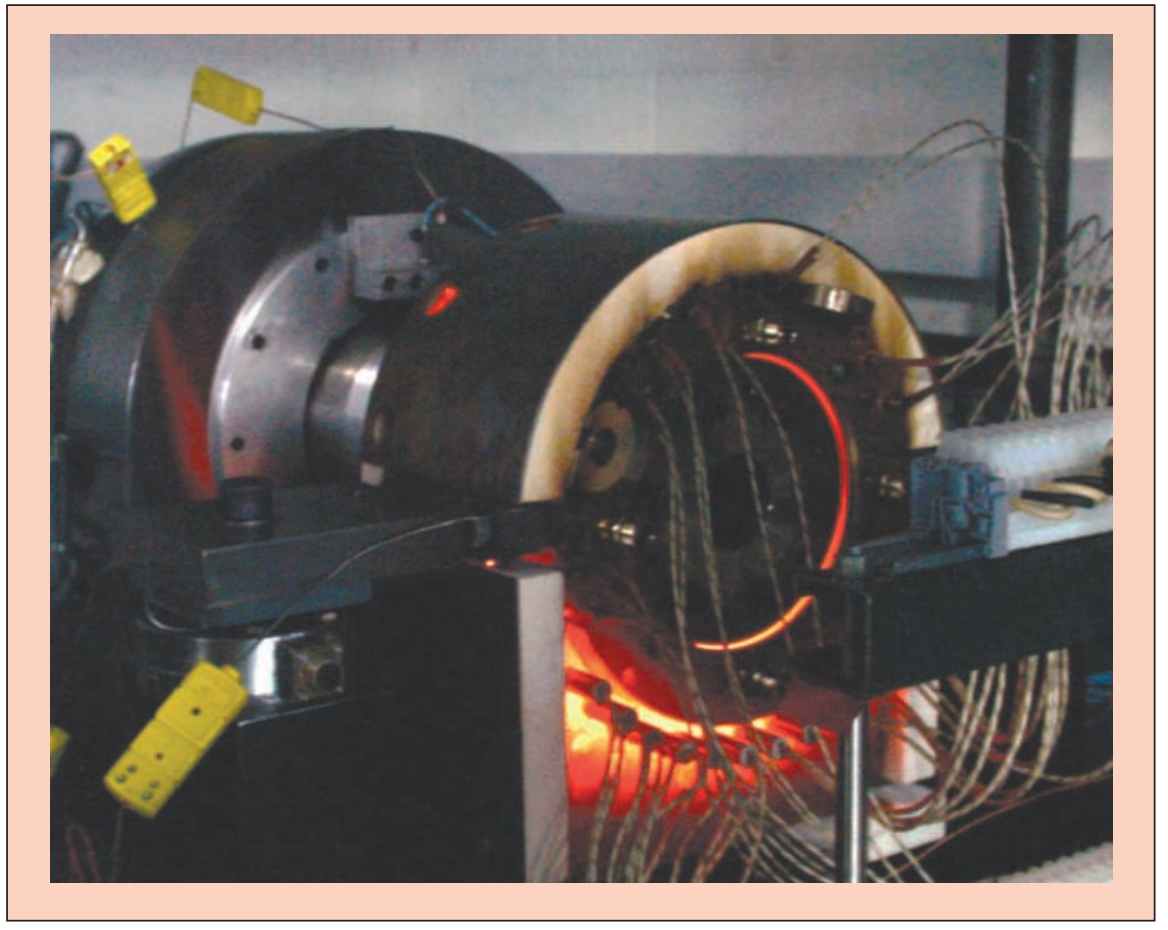

The Hybrid Foil Magnetic Bearing was photographed in operation at a speed of 15,000 rpm at a temperature of $1,200^{\circ} \mathrm{F}\left(>649^{\circ} \mathrm{C}\right)$ 